\title{
Nota Científica / Short Communication \\ Diagnóstico do conhecimento da biodiversidade de plantas vasculares nas unidades de conservação do estado de São Paulo
}

\author{
Diagnosis of biodiversity knowledge of vascular plants in protected areas \\ of São Paulo state
}

Matheus Colli-Silva ${ }^{1,4,5}$, Natália Macedo Ivanauskas ${ }^{2}$ \& Flaviana Maluf Souza $^{3}$

\begin{abstract}
Resumo
Com base em dados publicados sobre a ocorrência de espécies de plantas vasculares no estado de São Paulo, avaliamos a performance das unidades de conservação (UCs) na proteção dessas espécies, considerando especialmente aquelas endêmicas do estado e as ameaçadas de extinção. Das 648 espécies de samambaias e licófitas no estado, 71\% ocorrem em UCs, 10 são endêmicas de São Paulo e 30 estão ameaçadas de extinção. Pouco menos da metade das espécies de samambaias e licófitas ameaçadas não foram registradas em UCs, e três são endêmicas do estado. Para as plantas com semente, das 8.521 espécies registradas, 48\% ocorrem em UCs, 361 são endêmicas do estado e 676 estão ameaçadas de extinção. Entretanto, 65\% das espécies ameaçadas estão desprotegidas, e 51 destas são endêmicas do estado, a maioria nativa da Mata Atlântica. Muitas UCs ainda possuem grande lacuna de conhecimento sobre a flora, de esforço de coleta de material botânico e de registro em herbários, com destaque para as Reservas Particulares do Patrimônio Natural (RPPNs). Nossos dados reforçam a necessidade de ampliação de coletas botânicas em UCs, bem como a criação de novas áreas no estado, a fim de proteger espécies ameaçadas e endêmicas da flora paulista.
\end{abstract}

Palavras-chave: áreas protegidas, espécies ameaçadas, espécies endêmicas, flora paulista.

\begin{abstract}
Based on published data about occurrence of vascular plant species of São Paulo state, we analyzed the role of several protected areas (UCs) in the protection of these species, especially considering endemic or threatened ones. Of 648 ferns and lycophytes in the state, $71 \%$ occur in protected areas, 10 are endemics to São Paulo and 30 are threatened. Just under half of the threatened species of ferns and lycophytes were not registered in UCs, and three of these are endemic to the state. For seed plants, of 8,521 species recorded, $48 \%$ occur in UCs, 361 are endemics to the state and 676 are threatened. However, $65 \%$ of the threatened species are unprotected, and 51 of those are endemic to the state, most of them native to Atlantic Forest. Several UCs still have large lack of knowledge about their flora, of collection effort of botanical material and of record in herbaria, with particular emphasis on Private Natural Heritage Reserves (RPPNs). Our data reinforce the need for expansion of botanical collections in UCs, as well as the creation of new areas in the state, in order to protect threatened and endemic species of São Paulo flora. Key words: protected areas, threatened species, endemic species, São Paulo state flora.
\end{abstract}

Veja material suplementar em <https://doi.org/10.6084/m9.figshare.9894095.v1>

${ }^{1}$ Universidade de São Paulo, Depto. Botânica, Inst. Biociências, R. do Matão 277, 05508-090, São Paulo, SP, Brasil.

${ }^{2}$ Instituto Florestal, Seção de Ecologia Florestal, R. do Horto 931, 02377-000, São Paulo, SP, Brasil.

${ }^{3}$ Instituto Agronômico de Campinas, Centro de Análise e Pesquisa Tecnológica do Agronegócio de Horticultura, Av. Doutor Theodureto de Almeida Camargo 1500, 13075-630, Campinas, SP, Brasil.

${ }^{4}$ ORCID: <https://orcid.org/0000-0001-7130-3920>

${ }^{5}$ Autor para correspondência: matheus.colli.silva@usp.br 
As unidades de conservação (UCs) são áreas delimitadas para a proteção da biodiversidade, dos seus serviços e dos processos ecossistêmicos. São elementos importantes do ponto de vista da conservação da natureza, a qual vem sendo transformada pela ação antrópica nas últimas décadas (Gaston et al. 2008). Diante disso, diferentes entidades políticas, no nível estadual, nacional ou internacional, estabeleceram metas e compromissos que têm como objetivo nortear planos de ação direcionados para a conservação ou se baseiam em acordos ou compromissos internacionais. Exemplos importantes no Brasil são o Decreto $\mathrm{n}^{\circ} 4.339$ de 22 de agosto de 2002 e Lei $\mathrm{n}^{\circ} 13.123$ de 20 de maio de 2015, que delimitam aspectos da Política Nacional de Biodiversidade, bem como Weigrand-Junior et al. (2011), que discutem as Metas de Aichi definidas na Convenção da Diversidade Biológica.

O Brasil, que é um dos países com maior riqueza específica de plantas do mundo, bem como de espécies endêmicas (Brooks et al. 2002; Forzza et al. 2012; Ulloa-Ulloa et al. 2017), tem um papel importante no estabelecimento e na participação desses compromissos. Até 2020, o país se comprometeu a conhecer e descrever boa parte de sua biodiversidade, especialmente aquela presente em áreas de particular interesse para a conservação da natureza, como as unidades de conservação (Dias \& Hoft 2013). Além disso, comprometeu-se também a prevenir a extinção de mais espécies, estabelecendo novas políticas públicas conservacionistas (Weigrand-Junior et al. 2011).

Por um lado, o cumprimento de algumas dessas metas referentes à conservação da flora teve um avanço notável. O Brasil produziu um checklist de todas as suas espécies de plantas, algas e fungos do país, e hoje caminha para a elaboração de uma flora completa e acessível para todos os seus gêneros conhecidos até 2020 (Forzza et al. 2012). Além disso, alguns domínios fitogeográficos brasileiros possuem avanço notável na sua conservação, decorrente sobretudo da criação de uma série de unidades de conservação nessas regiões e da redução significativa na taxa de desmatamento e fragmentação em domínios como Amazônia e Mata Atlântica (Dias \& Hoft 2013).

Por outro lado, muitas outras metas ainda não foram cumpridas ou possuem avanços limitados, seja porque são muito ambiciosas e precisam de um esforço muito grande para serem inteiramente cumpridas num curto prazo, seja por conta de uma série de falhas no seu cumprimento e no estabelecimento de medidas de ação (Dias \& Hoft 2013). Aparentemente, a eficiência das UCs na proteção da biodiversidade do Brasil ainda conta com uma série de lacunas (Rodrigues et al. 2004; Colli-Silva et al. 2016; Oliveira et al. 2017) e muitas espécies ainda não possuem estado de conservação atribuído (Dias \& Hoft 2013), o que distancia o país de seus compromissos internacionalmente anunciados.

Diferentes produções técnicas e científicas são utilizadas como subsídios à conservação da biodiversidade vegetal no Brasil. Exemplos são a sistematização e criação de catálogos florísticos (Wanderley et al. 2005, 2011; Prado \& Hirai 2011; Forzza et al. 2012), estudos evolutivos (e.g., Carnaval \& Moritz 2008; Ribeiro et al. 2010), trabalhos de avaliação de serviços ecossistêmicos (Tambosi et al. 2014) e estabelecimento de listas vermelhas de espécies ameaçadas em diferentes escalas espaciais (Mamede et al. 2007; MMA2014; IUCN 2014). Em estudo recente sobre a ocorrência de espécies de plantas vasculares no estado de São Paulo (Colli-Silva et al. 2016), foi observado que as unidades de conservação de proteção integral e as RPPNs (Reservas Particulares do Patrimônio Natural) abrigam, atualmente, apenas cerca de $60 \%$ das espécies do estado, alertando sobre a necessidade de se intensificar o esforço de coleta nas unidades de conservação no estado, uma vez que muitas delas não estão bem amostradas (ColliSilva et al. 2016; Oliveira et al. 2017).

Um dos resultados desse estudo consiste em uma matriz que apresenta a ocorrência de mais de 4,8 mil espécies de plantas vasculares, incluindo as ameaçadas, quase ameaçadas e extintas, em UCs do território paulista. O trabalho consistiu no levantamento das plantas vasculares presentes nas unidades de conservação do estado, através dos dados presentes nas coleções de herbários disponibilizadas em grandes bases de dados virtuais (sobretudo no Herbário Virtual da Flora e dos Fungos, ICNT). Os autores avaliaram quais dos registros tinham como localização uma unidade de conservação, e com base nisso construíram a matriz de ocorrência das espécies para o estado (ver mais detalhes em Colli-Silva et al. 2016). Essa matriz permite avaliar o status quo da conservação de espécies de plantas no estado de São Paulo no que diz respeito à ocorrência de espécies ameaçadas, quase ameaçadas e extintas ou endêmicas no interior das UCs, algo que não foi realizado pelos autores no estudo supracitado. Diante disso, no 
presente estudo exploramos essas informações para avaliar mais a fundo o papel das unidades de conservação na proteção da biodiversidade vegetal, relacionando aspectos de (i) riqueza de espécies no estado; (ii) ocorrência nas unidades de conservação; e (iii) ameaça de extinção e endemismo no estado. Procuramos, com esta análise, responder: (1) qual a proporção da flora conhecida em UCs do estado de São Paulo; e (2) qual a proporção de espécies ameaçadas e endêmicas em UCs paulistas. Acreditamos que responder a essas questões poderá subsidiar o estabelecimento de políticas públicas voltadas para a conservação da flora paulista.

Para avaliar quais espécies de plantas vasculares ocorrem no estado de São Paulo, utilizamos os registros de herbário oriundos do REFLORA/CNPq (Projeto "Plantas do Brasil: Resgate Histórico e Herbário Virtual para o Conhecimento e Conservação da Flora Brasileira") e do Herbário Virtual da Flora e dos Fungos (INCT 2014), sendo os registros de Prado et al. (2015) referentes às samambaias e licófitas e BFG (2015) referentes às plantas com semente (gimnospermas e angiospermas). Estes checklists de espécies são derivados da ação conjunta de centenas de especialistas em taxonomia de várias instituições de pesquisa, e apresenta dados referentes à ocorrência e características das espécies de plantas, inclusive sobre distribuição e endemismo.

Para a consulta sobre as espécies ameaçadas, utilizamos dados da versão mais recente da lista vermelha mais recente publicada pela Secretaria do Meio Ambiente do Estado de São Paulo (SMA 2016), que apresenta critérios específicos para esse nível de escala espacial. Essa lista se baseia em critérios internacionais de elaboração de categorias de ameaça (critérios da International Union for Conservation of Nature - IUCN; ver Souza et al. 2007). No entanto, especificamente para a lista vermelha do estado de São Paulo, a inclusão de espécies e a atribuição da sua respectiva categoria de ameaça também são dependentes de uma combinação de critérios baseados nas informações sobre distribuição e ocorrência das espécies (ColliSilva et al. 2016). Essa versão da lista vermelha já integra os resultados do recente trabalho de Colli-Silva et al. (2016), que revisaram os critérios de ameaça das espécies com relação à ocorrência exclusiva ou desconhecida no interior de unidades de conservação.

Tanto os dados sobre espécies de plantas vasculares disponibilizados pelos checklists como a lista de espécies ameaçadas da SMA estão disponíveis para download. Os dados referentes ao REFLORA foram filtrados e sistematizados, de maneira que somente registros referentes ao estado de São Paulo permanecessem. Consideramos na análise apenas unidades de conservação de proteção integral (i.e. Parques Nacionais, Parques Estaduais, Estações Ecológicas, Reservas Biológicas, Monumentos Naturais e Refúgios de Vida Silvestre), e as Reservas Particulares do Patrimônio Natural (RPPNs), para corresponder aos critérios definidos pela lista vermelha da Secretaria do Meio Ambiente do Estado de São Paulo e aos usados no trabalho de Colli-Silva et al. (2016). Essas áreas, daqui em diante, serão as referidas como "unidades de conservação".

Os dados referentes às espécies ameaçadas também foram organizados quanto ao fato de estarem ameaçadas, quase ameaçadas ou extintas (VU - Vulnerável; EN - Em Perigo; CR - Em Perigo Crítico; EW - Extinto na Natureza; EX - Extinto), bem como quanto ao endemismo ou não no estado de São Paulo. Para sintetizar essas informações, fizemos diagramas com a relação de espécies endêmicas, protegidas e ameaçadas para cada um dos dois grandes grupos analisados. As Tabelas S1 e S2 (disponibilizadas no material suplementar <https://doi.org/10.6084/ m9.figshare.9894095.v1>), apresentam todos os dados por família, de espécies endêmicas do estado, ameaçadas de extinção e as que ocorrem no interior das unidades de conservação do estado.

Das 648 espécies de samambaias e licófitas registradas no estado de São Paulo, 71\% (464) ocorreram no interior de unidades de conservação, sendo 10 delas endêmicas do estado, e 30 ameaçadas de extinção. Das espécies ameaçadas, 43\% (13) não ocorreram no interior dessas áreas protegidas (Fig. 1). Destacamos Isoetes bradei Herter (Isoetaceae, categorizada como "Extinta" pelo SMA 2016, mas como "Em Perigo" pela IUCN), Ceradenia glaziovii (Baker) Labiak (Polypodiaceae) e Thelypteris multigemmifera Salino (Thelypteridaceae) (ambas classificadas como "Em Perigo"), que são endêmicas do estado de São Paulo e ameaçadas de extinção, mas que ainda não foram registradas no interior de nenhuma área protegida do estado. Por serem ameaçadas de extinção e endêmicas do estado, assume-se que essas são as espécies mais prioritárias do ponto de vista da conservação.

Com relação às plantas com semente, das 8.521 espécies descritas no estado, 48\% (4.104) foram registradas no interior de UCs. Existem 

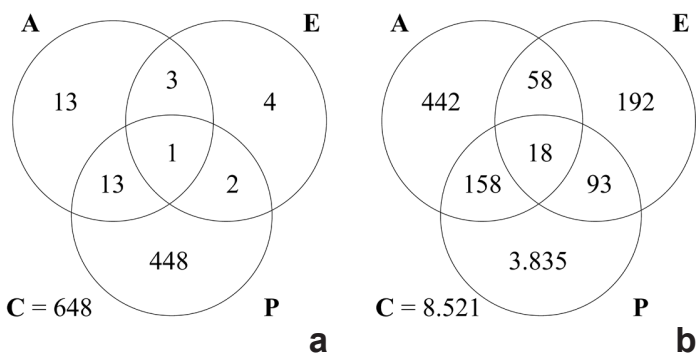

Figura 1 - a-b. Número de espécies de plantas vasculares conhecidas, protegidas por UCs ou ameaçadas de extinção no estado de São Paulo - a. samambaias e licófitas; b. plantas com semente. $(\mathrm{C}=$ todas as espécies que ocorrem no estado de São Paulo; $\mathrm{A}=$ espécies ameaçadas de extinção; $\mathrm{E}=$ espécies endêmicas do estado de São Paulo; $\mathrm{P}=$ espécies protegidas, i.e. com ao menos um registro no interior de pelo menos uma UC do estado).

Figure 1 - a-b. Number of known, protected (occurring inside a protected area) and threatened vascular plant species in São Paulo state - a. ferns and lycophytes; b. seed plants. $(\mathrm{C}=$ all plants occurring in São Paulo state; $\mathrm{A}=$ threatened species; $\mathrm{E}=$ species endemic to São Paulo state; $\mathrm{P}=$ protected species, i.e. with at least one record within any protected area of the state).
361 espécies endêmicas do estado de São Paulo, mas somente $33 \%$ destas (120) estão no interior de UCs. Com relação às espécies ameaçadas, das 676 presentes na lista vermelha, 65\% (442) delas não apresentam registros no interior de UCs. Dessas 442 espécies ameaçadas e desprotegidas, 58 são endêmicas do estado de São Paulo; ou seja, mais de $30 \%$ das espécies endêmicas do estado não ocorrem no interior das UCs delimitadas (Fig. 1; Tab. 1) e merecem especial atenção do ponto de vista de direcionamento de políticas públicas de conservação. Apesar de o Cerrado paulista ser o domínio mais ameaçado (Durigan 2006), a maioria das espécies dessa tabela são naturais da Mata Atlântica, o que também exige planos de ação mais efetivos na conservação da biodiversidade endêmica e ameaçada deste domínio. Para as samambaias e licófitas, 17 das 30 famílias possuem todas as suas espécies no interior das UCs. Para as plantas com semente, a situação é pior: das 201 famílias, apenas 40 possuem todas as suas espécies no interior de UCs. Avaliando apenas o universo das espécies ameaçadas de plantas vasculares,

Tabela 1 - Espécies de plantas com semente ameaçadas de extinção (ou extintas) e endêmicas do estado de São Paulo, mas que não possuem registros conhecidos no interior de ao menos uma unidade de conservação do território paulista. Categorias de ameaça: $\mathrm{CR}=$ Em Perigo Crítico; $\mathrm{EN}=$ Em Perigo; $\mathrm{EX}=$ Extinta; $\mathrm{VU}=$ Vulnerável.

Table 1 - Seed plants species that are threatened (or extinct) and endemic to São Paulo state, but do not have any register inside at least one protected area of São Paulo. Threat categories: $\mathrm{CR}=$ Critically Endangered; EN = Endangered; EX = Extinct; VU = Vulnerable.

\begin{tabular}{llcc}
\hline Família & Espécie & Domínio fitogeográfico & $\begin{array}{c}\text { Categoria de ameaça } \\
\text { (SMA 2016) }\end{array}$ \\
\hline Annonaceae & Annona ubatubensis (Maas \& Westra) H.Rainer & Mata Atlântica & EN \\
Apocynaceae & Macroditassa marianae Fontella \& M.V.Ferreira & Mata Atlântica & EN \\
Araceae & Anthurium ameliae Nadruz \& Cath. & Mata Atlântica & VU \\
Araceae & Asterostigma cubense (A.Rich.) & Mata Atlântica & VU \\
& K.Krause ex Bogner & & \\
Araceae & Philodendron simonianum Sakur. & Mata Atlântica & VU \\
Begoniaceae & Begonia handroi Brade & Mata Atlântica & EX \\
Begoniaceae & Begonia larorum L.B.Sm. \& Wassh. & Mata Atlântica & EX \\
Bromeliaceae & Aechmea wittmackiana (Regel) Mez & Mata Atlântica & VU \\
Bromeliaceae & Neoregelia nivea Leme & Mata Atlântica & VU \\
Bromeliaceae & Nidularium corallinum (Leme) Leme & Mata Atlântica & EN \\
Bromeliaceae & Nidularium rolfianum Leme & Mata Atlântica & VU \\
Chrysobalanaceae & Couepia meridionalis Prance & Mata Atlântica & EX \\
Ericaceae & Gaultheria sleumeriana Kin.-Gouv. & Mata Atlântica & EN \\
\hline
\end{tabular}




\begin{tabular}{|c|c|c|c|}
\hline Família & Espécie & Domínio fitogeográfico & $\begin{array}{l}\text { Categoria de ameaça } \\
\text { (SMA 2016) }\end{array}$ \\
\hline Erythroxylaceae & Erythroxylum cyclophyllum O.E.Schulz & Mata Atlântica & EX \\
\hline Fabaceae & $\begin{array}{l}\text { Chamaecrista atroglandulosa } \\
\text { (Taub. ex Harms) H.S.Irwin \& Barneby }\end{array}$ & Mata Atlântica & EX \\
\hline Gesneriaceae & Nematanthus mattosianus (Handro) H.E.Moore & Mata Atlântica & VU \\
\hline Gesneriaceae & Sinningia araneosa Chautems & Cerrado & VU \\
\hline Gesneriaceae & Sinningia iarae Chautems & Mata Atlântica & $\mathrm{EN}$ \\
\hline Gesneriaceae & Sinningia insularis (Hoehne) Chautems & Mata Atlântica & $\mathrm{EN}$ \\
\hline Gesneriaceae & Sinningia micans (Fritsch) Chautems & Mata Atlântica & $\mathrm{EN}$ \\
\hline Gesneriaceae & Sinningia piresiana (Hoehne) Chautems & Mata Atlântica & $\mathrm{EN}$ \\
\hline Lamiaceae & Aegiphila capitata Moldenke & Mata Atlântica & EX \\
\hline Lamiaceae & Hyptis lobata A.St.-Hil. ex Benth. & Mata Atlântica & EX \\
\hline Lamiaceae & Hyptis riparia Harley & Cerrado, Mata Atlântica & EX \\
\hline Lauraceae & Aiouea bracteata Kosterm. & Cerrado, Mata Atlântica & VU \\
\hline Lauraceae & Ocotea felix Coe-Teix. & Mata Atlântica & EN \\
\hline Lauraceae & Ocotea inhauba Coe-Teixeira & Mata Atlântica & EX \\
\hline Lauraceae & Persea rigida Nees \& Mart. & Mata Atlântica & $\mathrm{EN}$ \\
\hline Malvaceae & Abutilon nigricans G.L.Esteves \& Krapov. & Mata Atlântica & $\mathrm{CR}$ \\
\hline Orchidaceae & Epidendrum infaustum Rchb.f. & Mata Atlântica & EX \\
\hline Orchidaceae & Habenaria galeandriformis Hoehne & Mata Atlântica & EX \\
\hline Orchidaceae & Habenaria jordanensis (J.E.Leite) Garay & Mata Atlântica & EX \\
\hline Orchidaceae & Habenaria novaesii Edwall \& Hoehne & Mata Atlântica & VU \\
\hline Orchidaceae & Macradenia paulensis Cogn. & Mata Atlântica & EX \\
\hline Orchidaceae & Miltonia kayasimae Pabst & Mata Atlântica & $\mathrm{CR}$ \\
\hline Orchidaceae & Octomeria hoehnei Schltr. & Mata Atlântica & EX \\
\hline Orchidaceae & Octomeria wilsoniana Hoehne & Mata Atlântica & EX \\
\hline Orchidaceae & Phymatidium vogelii Pabst & Mata Atlântica & VU \\
\hline Orchidaceae & Scuticaria itirapinensis Pabst & Cerrado & VU \\
\hline Oxalidaceae & Oxalis arachnoidea Progel & Mata Atlântica & $\mathrm{CR}$ \\
\hline Passifloraceae & Passiflora ischnoclada Harms & Mata Atlântica & $\mathrm{CR}$ \\
\hline Piperaceae & Peperomia cooperi C.DC. & Mata Atlântica & EX \\
\hline Piperaceae & Peperomia guarujana C.DC. & Mata Atlântica & EX \\
\hline Piperaceae & Peperomia hemmendorffii Yunck. & Mata Atlântica & EX \\
\hline Piperaceae & Piper amparoense Yunck. & Mata Atlântica & EX \\
\hline Piperaceae & Piper hoehnei Yunck. & Mata Atlântica & EX \\
\hline Piperaceae & Piper kuhlmannii Yunck. & Mata Atlântica & EX \\
\hline Piperaceae & Piper loefgrenii Yunck. & Mata Atlântica & EX \\
\hline Piperaceae & Piper oblancifolium Yunck. & Mata Atlântica & EX \\
\hline
\end{tabular}




\begin{tabular}{llcc}
\hline Familia & Espécie & Domínio fitogeográfico & $\begin{array}{c}\text { Categoria de ameaça } \\
\text { (SMA 2016) }\end{array}$ \\
\hline Poaceae & Chusquea erecta L.G.Clark & Mata Atlântica & EN \\
Poaceae & Merostachys bradei Pilg. & Mata Atlântica & EX \\
Poaceae & Merostachys brevigluma Send. & Mata Atlântica & EN \\
Poaceae & Merostachys polyantha McClure & Mata Atlântica & EN \\
Rubiaceae & Chomelia modesta (Standl.) Steyerm. & Mata Atlântica & EX \\
Rubiaceae & Galianthe souzae E.L.Cabral \& Bacigalupo & Cerrado & EN \\
Rubiaceae & Psychotria loefgrenii Standl. & Mata Atlântica & EN \\
Solanaceae & Solanum spissifolium Sendtn. & Mata Atlântica & EX \\
Xyridaceae & Xyris uninervis Malme & Cerrado & VU \\
\hline
\end{tabular}

apenas $27 \%$ das 706 espécies (190) possuem registros no interior de UCs. Mesmo considerando as diferentes categorias de ameaça, pelo menos $50 \%$ das espécies não foram coletadas no interior de UCs, o que agrava a situação do ponto de vista de conservação (Tab. 2).

Ou seja, além de as espécies ameaçadas não serem bem representadas na rede de unidades de conservação do estado de São Paulo, o esforço de coleta também não é homogêneo. Embora tenha sido estipulada uma meta de registro de ao menos uma exsicata por quilômetro quadrado (Durigan $e t$ al. 2008), nossos dados não revelam esse panorama (Fig. 2), e estima-se que no estado de São Paulo existam somente 0,25 registros georreferenciados de plantas com semente por quilômetro quadrado (Cielo-Filho et al. 2009). Oliveira et al. (2016) também destacam o forte viés de coleta que existe no Brasil, pois o maior maior número de coletas se concentra nas áreas de fácil acesso ou em locais onde há melhor infraestrutura para receber a visita dos pesquisadores, o que inclui várias unidades de conservação. Além disso, o viés de coleta também deve estar associado ao viés taxonômico, já que certas famílias são mais amostradas que outras (Fig. 3), seja porque algumas são mais facilmente coletáveis, amostráveis, acessíveis, estudadas ou de mais fácil identificação que outras.

Dentre as 115 UCs consideradas neste estudo, 64 se encontram abaixo da meta prevista, $\mathrm{e}$, independente da categoria de unidade de conservação, todas possuem no mínimo $30 \%$ de seus territórios com menos de um registro ou espécie por quilômetro quadrado, ou não possuem dados. A situação é particularmente preocupante para RPPNs, em que a maioria sequer possui material depositado em herbários (Fig. 2), e cujo acesso pode se tornar difícil, pois depende da autorização do proprietário do terreno privado onde se insere a unidade de conservação (Mesquita 2004).

Estudos com outros grupos de seres vivos e escalas espaciais também apresentaram situação semelhante ou pior ao aqui apresentado (ver, dentre outros, Fearnside \& Ferraz 1995; Rodrigues et al. 2004; Laurance et al. 2012; Watson et al. 2014; Oliveira et al. 2017). Destacamos o estudo de Oliveira et al. (2017), que considerou não só grupos de plantas, mas também animais, e cujo nível espacial de análise abrangeu todo o território brasileiro. Neste trabalho, $70 \%$ das unidades de conservação possuem menos de 0,01 registros de espécies por quilômetro quadrado, e cerca da metade das UCs do Brasil sequer possuem registros em herbários, museus ou outras coleções biológicas.

Além disso, outros estudos que avaliam a performance e o potencial das áreas protegidas nas regiões tropicais (Laurance et al. 2012; Watson et al. 2014) alertam para a necessidade do financiamento e planos de ação ainda deficientes em muitos países compromissados com as metas internacionais de conservação da natureza. O Brasil está incluso nessa lista de países que perderam grande parte da sua vegetação remanescente nas últimas décadas, bem como grande parte da biodiversidade nas suas unidades de conservação. Para São Paulo, o padrão de distribuição de registros de plantas em unidades de conservação foi similar ao panorama do território brasileiro como um todo, considerando seus diferentes domínios fitogeográficos e independente do número de 
Tabela 2 - Porcentagem de espécies ameaçadas de (I) samambaias e licófitas e (II) plantas com semente, considerando a ocorrência da espécie no interior de unidades de conservação, seu endemismo no estado de São Paulo, bem como as categorias de ameaça. $\mathrm{A}$ = espécies ameaçadas de extinção, quase ameaçadas ou extintas; $\mathrm{E}=$ endêmicas do estado de São Paulo; P = espécies com maior grau de proteção, pois foram registradas em unidades de conservação. As combinações de letras representam os seguintes agrupamentos: AE = espécies ameaçadas de extinção, quase ameaçadas ou extintas, endêmicas do estado de São Paulo e que não possuem registros no interior de UCs; AE = espécies ameaçadas de extinção ou extintas, não endêmicas do estado de São Paulo e que não possuem registros no interior de UCs; PA = espécies ameaçadas de extinção ou extintas, não endêmicas do estado de São Paulo e que possuem registros no interior de UCs; PAE = espécies ameaçadas de extinção ou extintas, endêmicas do estado de São Paulo e que possuem registros no interior de UCs. Critérios de ameaça: CR = Em Perigo Crítico; EN = Em Perigo; $\mathrm{EW}=$ Extinta na Natureza; $\mathrm{EX}=$ Extinta; $\mathrm{VU}=$ Vulnerável; NT = Quase Ameaçada.

Table 2 - Percentage of threatened species of (I) ferns and lycophytes and (II) seed plants, considering the occurrence of those species inside protected areas, their endemism in the state of São Paulo, as well as threat categories. $A=$ threatened, near threatened or extinct species; $\mathrm{E}=$ species endemic to the state of São Paulo; $\mathrm{P}=$ species with higher degree of protection since there are records inside UCs. Letter combinations represent the following groupings: $\mathrm{AE}=$ threatened or extinct species, endemic to the state of São Paulo and that have no records inside UCs; PA = threatened or extinct species, not endemic to the state of São Paulo and that have records inside UCs; $\mathrm{PAE}=$ threatened or extinct species, endemic to the state of São Paulo and that have records inside UCs. Threat criteria: $\mathrm{CR}=\mathrm{Critically}$ Endangered; EN = Endangered; EW = Extinct in the Wild; EX = Extinct; VU = Vulnerable; NT = Near Threatened.

\begin{tabular}{lcccc}
\hline & \multicolumn{2}{c}{ I - samambaias e licófitas } & AE & PA \\
Rótulos de linha & A & PAE & 0 & 0 \\
\hline NT $(\mathrm{N}=0)$ & 0 & 0 & 0 & 4 \\
VU $(\mathrm{N}=9)$ & 5 & 0 & 2 & 9 \\
EN $(\mathrm{N}=19)$ & 7 & 1 & 0 & 0 \\
CR $(\mathrm{N}=0)$ & 0 & 0 & 0 & 0 \\
EW $(\mathrm{N}=0)$ & 0 & 0 & 1 & 0 \\
EX $(\mathrm{N}=2)$ & 1 & 0 & 3 & 13 \\
\hline Todas $(\mathrm{N}=30)$ & 13 & 1 & & PAE \\
\hline & $\mathbf{I I}-$ plantas com semente & $\mathbf{A}$ & 0 \\
Rótulos de linha & 1 & 7 & 2 & 5 \\
\hline NT $(\mathrm{N}=10)$ & 15 & 130 & 47 & 5 \\
VU $(\mathrm{N}=197)$ & 8 & 115 & 35 & 2 \\
EN $(\mathrm{N}=163)$ & 4 & 27 & 15 & 0 \\
CR $(\mathrm{N}=48)$ & 2 & 6 & 3 & 6 \\
EW $(\mathrm{N}=11)$ & 28 & 156 & 56 & 18 \\
EX $(\mathrm{N}=246)$ & 58 & 441 & 158 & \\
\hline Todas $(\mathrm{N}=676)$ & & & & \\
\hline
\end{tabular}

espécies em cada domínio. Portanto, ainda são necessários esforços direcionados para a criação de novas unidades e novas medidas de proteção para as UCs paulistas, sobretudo considerando as espécies ameaçadas, o que também irá contribuir para a melhoria das métricas de nacionais.

Com base nos dados aqui apresentados e nos disponíveis na literatura, reforçamos também a necessidade do fomento à pesquisa direcionada para inventários florísticos nos remanescentes paulistas, coordenado por equipe eficaz no inventário de trechos ainda não explorados. Reiteramos que incentivos a essas expedições botânicas apresentam alta relação custo-benefício para os planos de manejo dessas unidades, ampliando assim o seu papel na conservação da biodiversidade e no 


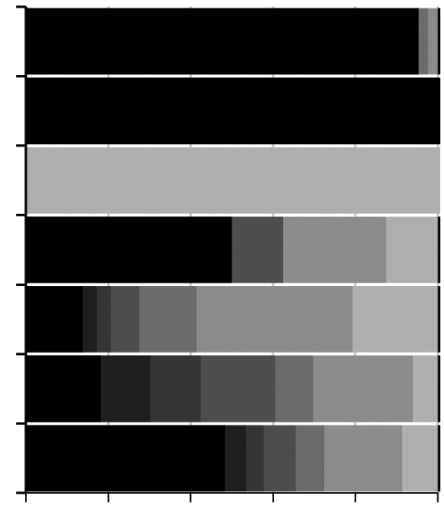

$\operatorname{RPPN}(\mathrm{N}=43)$

$\mathrm{MN}(\mathrm{N}=1)$

$\operatorname{RVS}(\mathrm{N}=1)$

$\mathrm{RB}(\mathrm{N}=8)$

$\mathrm{EE}(\mathrm{N}=29)$

$\mathrm{PE} / \mathrm{PN}(\mathrm{N}=33)$

Todas $(\mathrm{N}=115)$

$\begin{array}{llllll}0 \% & 20 \% & 40 \% & 60 \% & 80 \% & 100 \%\end{array}$

\section{a}

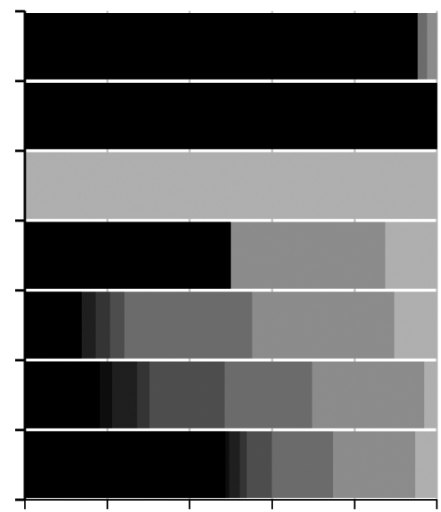

$\operatorname{RPPN}(\mathrm{N}=43)$

$\mathrm{MN}(\mathrm{N}=1)$

$\operatorname{RVS}(\mathrm{N}=1)$

$\mathrm{RB}(\mathrm{N}=8)$

$\mathrm{EE}(\mathrm{N}=29)$

PE/PN (N = 33)

Todas $(\mathrm{N}=115)$

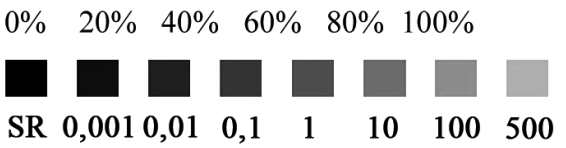

b

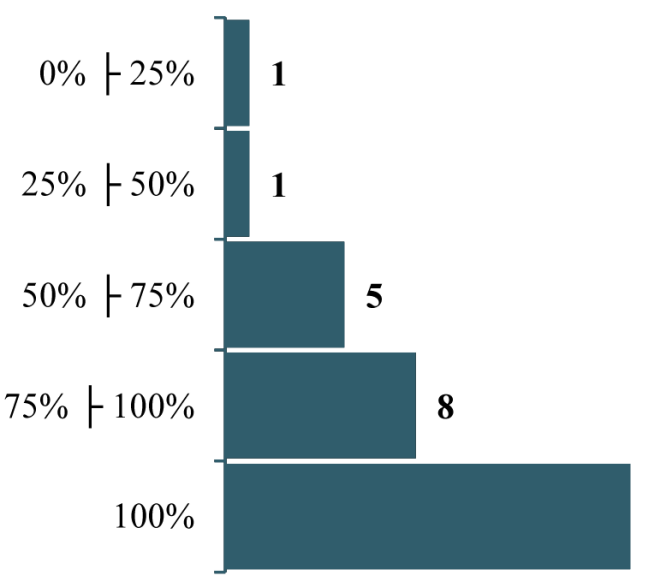

17

Famílias

a

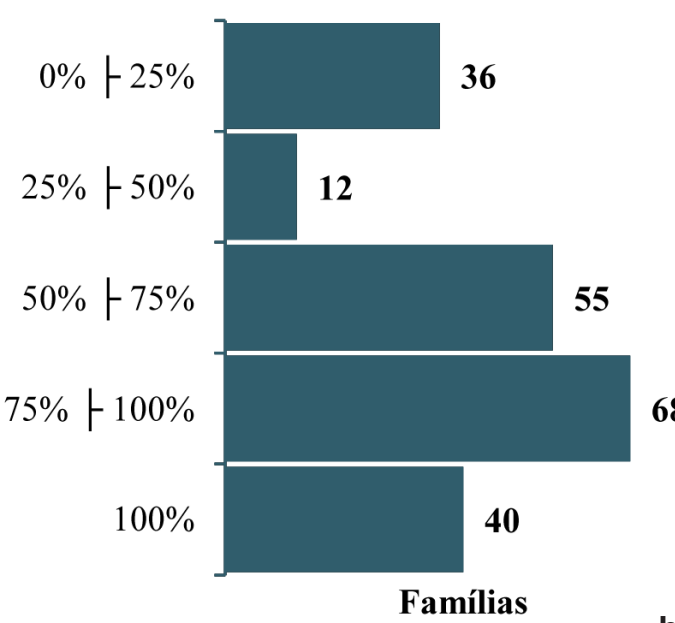

b
Figura 2 - a. Porcentagem de unidades de conservação do estado de São Paulo que possuem diferentes valores de densidade de registros (exsicatas $/ \mathrm{km}^{2}$ ) de plantas vasculares; b. porcentagem de UCs que possuem diferentes valores de densidade de espécies (espécies/ $\mathrm{km}^{2}$ ) de plantas vasculares. As UCs estão divididas quanto à sua categoria. RPPN = Reservas Particulares do Patrimônio Natural; $\mathrm{MN}=$ Monumento Natural; RVS = Reserva de Vida Silvestre; RB = Reserva Biológica; EE = Estação Ecológica; PE/PN = Parque Estadual/Nacional; $\mathrm{SR}=$ sem registro.

Figure 2 - a. Percent of protected areas of São Paulo state with different scores of record density (vouchers $/ \mathrm{km}^{2}$ ) of vascular plants; b. percent of protected areas with different scores of species density (species $/ \mathrm{km}^{2}$ ) of vascular plants. Protected areas are classified in RPPN = Private Reserve of Nature Patrimony; $\mathrm{MN}=$ Natural Monument; RVS = Wildlife Reserve; $\mathrm{RB}=$ Biological Reserve; $\mathrm{EE}$ $=$ Ecological Station; $\mathrm{PE} / \mathrm{PN}=$ National/State Park; $\mathrm{SR}=$ no data.
Figura 3 - a-b. Número de famílias de acordo com a porcentagem de espécies da família com registros em UCs - a. samambaias e licófitas; b. plantas com semente. A figura mostra que a maioria das famílias de samambaias e licófitas (17 famílias) possui todas as suas espécies $(100 \%)$ protegidas em UCs, enquanto para as plantas com semente, a maioria das famílias (68 famílias) possui entre 75 e 100\% das suas espécies protegidas.

Figure 3 - $a-b$. Number of families according to the fraction of all species of the family in protected areas of São Paulo state - a. ferns and lycophytes; b. seed plants. The figure show that most fern/lycophyte families (17 families) have all of their species $(100 \%)$ inside protected areas, whereas for seed plants, most families (68 families) have between 75 and $100 \%$ of their species protected. 
conhecimento da flora paulista. Nesse cenário, é importante priorizar inventários nas UCs cujo número de espécies da flora conhecida está abaixo do esforço amostral desejado (isto é, inferior a uma exsicata por quilômetro quadrado).

Com relação às populações de espécies ameaçadas de extinção, o padrão de coocorrência dessas espécies em determinadas áreas também é importantíssimo para a elaboração de planos de ação voltados para a reversão do grau de ameaça a que essas espécies estão sujeitas. Deve-se incentivar a ampliação de estudos sobre a flora nas unidades de conservação, os quais devem estar disponíveis para elaboração de planos de manejo, a fim de nortear as estratégias de conservação para populações ameaçadas de extinção.

As conclusões aqui apresentadas não são, portanto, restritas ao estado de São Paulo: muito do conhecimento da biodiversidade brasileira possui lacunas, com determinadas áreas mais coletadas, o que, independentemente do grupo taxonômico (Laurance et al. 2012; Watson et al. 2014; Oliveira et al. 2016), exige coleta mais intensa em áreas remotas (Tabarelli et al. 2005; Giulietti et al. 2005). Isso significa que ainda há necessidade de expedições para coleta de novos espécimes, a fim de ampliar o conhecimento sobre a flora paulista, mesmo num estado onde se concentra grande número de taxonomistas. Além disso, muitas das lacunas de amostragem podem ser não devido a uma lacuna de coleta, mas sim devido à não incorporação de material nos herbários, que são coleções importantes para a conservação da biodiversidade porque sistematizam os registros de ocorrência necessários para a acuraria da definição de categorias de ameaça e, consequentemente, de políticas públicas, mas cujas coleções no Brasil ainda precisam e podem ser expandidas (Schatz 2002; Funk 2009; Forzza et al. 2012).

\section{Agradecimentos}

Os autores agradecem ao CNPq (Conselho Nacional de Desenvolvimento Científico e Tecnológico), a bolsa de Iniciação Científica concedida ao primeiro autor em 2014-2015.

\section{Referências}

BFG - The Brazil Flora Group (2015) Growing knowledge: an overview of seed plant diversity in Brazil. Rodriguésia 66: 1085-1113.

Brasil (2002) Decreto n ${ }^{\circ} .4 .339$, de 22 de agosto de 2002. Institui princípios e diretrizes para a implementação da Política Nacional da Biodiversidade. Disponível em <http://www.planalto.gov.br/ccivil 03/ decreto/2002/D4339.htm>. Acesso em 12 julho 2017.

Brasil (2015) Lei n ${ }^{\circ}$. 13.123, de 20 de maio de 2015. Regulamenta o inciso II do $\S 1^{\circ}$ e o $\S 4^{\circ}$ do art. 225 da Constituição Federal, o Artigo 1, a alínea j do Artigo 8, a alínea c do Artigo 10, o Artigo 15 e os $\S \S 3^{\circ}$ e $4^{\circ}$ do Artigo 16 da Convenção sobre Diversidade Biológica, promulgada pelo Decreto $\mathrm{n}^{\circ}$ 2.519, de 16 de março de 1998; dispõe sobre o acesso ao patrimônio genético, sobre a proteção e o acesso ao conhecimento tradicional associado e sobre a repartição de benefícios para conservação e uso sustentável da biodiversidade; revoga a Medida Provisória n ${ }^{\circ}$ 2.186-16, de 23 de agosto de 2001; e dá outras providências. Disponível em $<\mathrm{http}$ ://www. planalto.gov.br/ccivil_03/_ato2015-2018/2015/lei/ 113123.htm>. Acesso em 12 julho 2017.

Brooks TM, Mittermeier RA, Mittermeier CG, Fonseca GAB, Rylands AB, Konstant WR, Flick P, Pilgrim J, Oldfield S, Magin G \& Hilton-Taylor C (2002) Habitat loss and extinction in the hotspots of biodiversity. Conservation Biology 16: 909-923.

Carnaval AC \& Moritz C (2008) Historical climate modeling predicts patterns of current biodiversity in the Brazilian Atlantic forest. Journal of Biogeography 35: 1187-1201.

Cielo-Filho R, Baitello JB, Pastore JA, Aguiar OT, Souza SCPM, Toniato MTZ, Lima CR \& Ribeiro AP (2009) Ampliando a densidade de coletas botânicas na região da bacia hidrográfica do Alto Paranapanema: caracterização florística da floresta estadual e da estação ecológica de Paranapanema. Biona Neotropica 9: 255-276.

Colli-Silva M, Bezerra TL, Franco GADC, Ivanauskas NM \& Souza FM (2016) Registros de espécies vasculares em unidades de conservação e implicações para a lista da flora ameaçada de extinção no estado de São Paulo. Rodriguésia 67: 405-425.

Dias BS \& Hoft R (2013) Challenges facing implementation of the Global Strategy for Plant Conservation in Brazil. In: Martinelli G \& Moraes MA (eds.) Red Book of Brazilian flora. Nacional Center for Flora Conservation, Rio de Janeiro. Pp. 26-39.

Durigan G (2006) Observations on the southern cerrados and their relationship with the core area. In: Pennington RT, Lewis GP \& Ratter JA (eds.) Neotropical savannas and seasonally dry forests: plant diversity, biogeography and conservation. Taylor \& Francis, London. Pp. 67-77.

Durigan G, Mamede MCH, Ivanauskas NM, Siqueira MF, Moura C, Barros F, Souza FM, Vilela FESP, Arzolla FARP, Franco GADC, Cordeiro I, Koch I, Baitello JB, Lombardi JA, Lima LR, Lohmann LG, Bernacci LC, Assis MA, Aidar MPM, Wanderley MGL, Toniato MTZ, Ribeiro M, Groppo M, 
Cavassan O, Sano PT, Rodrigues RR, Fichs TV \& Martins SE (2008) Fanerógamas. In: Rodrigues RR, Bononi VLR (orgs.) Diretrizes para a conservação e restauração da biodiversidade no estado de São Paulo. Instituto de Botânica/Imprensa Oficial do Estado de São Paulo, São Paulo. Pp. 104-109.

Fearnside PM \& Ferraz J (1995) A conservation gap analysis of Brazil's Amazonian vegetation. Conservation Biology 9: 1134-1147.

Forzza RC, Baumgratz JFA, Bicudo CEM, Canhos D, Carvalho Jr. AA, Nadruz-Coelho MA, Costa AF, Costa DP, Hopkins M, Leitman PM, Lohmann LG, Lughadha EN, Maia LC, Martinelli G, Menezes M, Morim MP, Peixoto AL, Pirani JR, Prado J, Queiroz LP, Souza S, Souza VC, Stehmann JR, Sylvestre LS, Walter BMT \& Zappi DC (2012) New Brazilian floristic list highlights conservation challenges. BioScience 62: 39-45.

Funk VA (2009) 100 uses of an herbarium. ASPT Newsletter 17: 17-19.

Gaston KJ, Jackson SF, Cantú-Salazar L \& Cruz-Piñon G (2008) The ecological performance of protected areas. Annual Review of Ecology, Evolution and Systematics 39: 93-113.

Giulietti AM, Harley RM, Queiroz LP, Wanderley MGL \& Berg CV (2005) Biodiversidade e conservação das plantas no Brasil. Megadiversidade 1: 52-61.

Hoft R \& Dias BFS (2013) Desafios para a implementação da estratégia global para a conservação de plantas no Brasil. In: Martinelli G \& Moraes MA (orgs.) Livro vermelho da Flora do Brasil. Instituto de Pequisas Jardim Botânico do Rio de Janeiro, Rio de Janeiro. Pp. 26-39.

INCT - Herbário Virtual da Flora e dos Fungos (2014) Disponível em $<$ http://inct.splink.org.br $>$. Acesso contínuo desde agosto 2017.

IUCN - International Union for Conservation of Nature (2014) Standards and petitions subcommittee. Guidelines for Using the IUCN Red List Categories and Criteria. Version 11. Disponível em $<$ http://www. iucnredlist.org/documents/RedListGuidelines.pdf $>$. Acesso em 11 maio 2016.

Laurance WF, Carolina D, Rendeiro J, Kalka M, Bradshaw CJA, Sloan SP, Laurance SG, Campbell M, Abernethy K, Alvarez P, Arroyo-Rodriguez V, Ashton P, Benítez-Malvido J, Blom A, Bobo KS, Cannon $\mathrm{CH}$, Cao M, Carroll R, Chapman C, Coates R, Cords M, Danielsen F, Dijn B, Dinerstein E, Donnelly MA, Edwards D, Edwards F, Farwig N, Fashing P, Forget P, Foster M, Gale G, Harris D, Harrison R, Hart J, Karpanty S, John W, Krishnaswamy J, Logsdon W, Lovett J, Magnusson W, Maisels F, Marshall AR, McClearn D, Mudappa D, Nielsen MR, Pearson R, Pitman N, van Weerd J, Plumptre A, Poulsen J, Quesada M, Rainey H, Robinson D, Roetgers C, Rovero F, Scatena F, Schulze C, Sheil D, Struhsaker T, Terborgh J, Thomas D, Timm R, Nicolas J, Vasudevan K,
Joseph S, Carlos J, Arroyo L, Ashton M, Auzel P, Babaasa D, Babweteera F, Baker P, Banki O, Bass M, Bila-Isia I, Blake S, Brockelman W, Brokaw N, Brühl CA, Bunyavejchewin S, Chao J, Chave J, Chellam R, Clark CJ, Clavijo J, Congdon R, Corlett R, Dattaraja HS, Dave C, Davies G, Beisigiel BM, Silva RNP, Di Fiore A, Diesmos A, Dirzo R, Doran-Sheehy D, Eaton M, Emmons L, Estrada A, Ewango C, Fedigan L, Feer F, Fruth B, Giacalone J, Goodale U, Goodman S, Guix JC, Guthiga P, Haber W, Hamer K, Herbinger I, Hill J, Huang Z, Fang I, Ickes K, Itoh A, Ivanauskas NM, Jackes B, Janovec J, Janzen D, Jiangming M, Jin C, Jones T, Justiniano H, Kalko E, Kasangaki A, Killeen T, King H, Klop E, Knott C, Koné I, Kudavidanage E, Lahoz J, Lattke J, Laval R, Lawton R, Leal M, Leighton M, Lentino M, Leonel C, Lindsell J, Ling-Ling L, Eduard K, Losos E, Lugo A, Lwanga J, Mack AL, Martins M, Scott W, McNab R, Montag L, Myers J, Nabe-Nielsen J, Nakagawa M, Nepal S, Norconk M, Novotny V, O'Donnell S, Opiang M, Ouboter P, Parker K, Parthasarathy N, Pisciotta K, Prawiradilaga D, Pringle C, Rajathurai S, Reichard U, Reinartz G, Renton K, Reynolds G, Reynolds V, Riley E, Rödel M, Rothman J, Round P, Sakai S, Sanaiotti T, Savini T, Schaab G, Seidensticker J, Siaka A, Silman MR, Smith TB, de SS, Sodhi N, Stanford C, Stewart K, Stokes E, Stoner KE, Sukumar R, Surbeck M, Tobler M, Tscharntke T, Turkalo A, Umapathy G, van $M$, Vega J, Venkataraman M, Venn L, Verea C, Volkmer C, Waltert M, Wang B, Watts D, Weber W, West P, Whitacre D, Whitney K, Wilkie D, Williams S, Wright DD, Wright P, Xiankai L, Yonzon P \& Zamzani F (2012) Averting biodiversity colapse in tropical forest protected areas. Nature 489: 290-294.

Mamede MCH, Souza VC, Prado J, Barros F, Wanderley MGL \& Rando JG (2007) Livro vermelho das espécies vegetais ameaçadas de extinção no estado de São Paulo. Instituto de Botânica, São Paulo. $165 \mathrm{p}$.

Mesquita CAB (2004) Breve histórico da conservação em terras privadas, do código florestal até as RPPNs. In: Mesquita CAB \& Vieira MCW (orgs.) RPPN - Reservas Particulares do Patrimônio Natural da Mata Atlântica. Conselho Nacional da Reserva da Biosfera da Mata Atlântica, São Paulo. Pp. 8-13.

MMA - Ministério do Meio Ambiente (2014) Portaria MMA n ${ }^{\circ} 443$, de 17 de dezembro de 2014. Lista Nacional Oficial de Espécies da Flora Ameaçadas de Extinção. Disponível em <http://cncflora.jbrj. gov.br/portal/static/pdf/portaria_mma_443_2014. pdf $>$. Acesso em 11 maio 2016.

Oliveira U, Paglia AP, Brescovit AD, Carvalho CJB, Silva DP, Rezende DT, Leite FSF, Batista JAN, Barbosa JPPP, Stehmann JR, Ascher JS, Vasconcelos MF, De Marco Jr. P, Löwenberg-Neto P, Dias PG, Ferro VG \& Santos AJ (2016) The strong influence of collection bias on biodiversity knowledge shortfalls 
of Brazilian terrestrial biodiversity. Diversity and Distributions 22: 1232-1244.

Oliveira U, Soares-Filho BS, Paglia AP, Brescovit AD, Carvalho CJB, Silva DP, Rezende DT, Leite FSF, Batista JAN, Barbosa JPPP, Stehmann JR, Ascher JS, Vasconselos MF, Marco P, Löwenberg-Neto P, Ferro VG \& Santos AJ (2017) Biodiversity conservation gaps in the Brazilian protected áreas. Scientific Reports 7: 9141.

Prado J \& Hirai RY (2011) Checklist das licófitas e samambaias do estado de São Paulo, Brasil. Biota Neotropica 11: 161-190.

Prado J, Sylvestre LS, Labiak PH, Windisch PG, Salino A, Barros ICL, Hirai RY, Almeida TE, Santiago ACP, Kieling-Rubio MA, Pereira AFN, Øllgaard B, Ramos CGV, Mickel JT, Dittrich VAO, Mynssen CM, Schwartsburd PB, Condack JPS, Pereira JBS \& Matos FB (2015) Diversity of ferns and lycophytes in Brazil. Rodriguésia 66: 1-11.

Ribeiro RA, Lemos-Filho JP, Ramos AC \& Lovato MB (2010) Phylogeography of the endangered rosewood Dalbergia nigra (Fabaceae): insights into the evolutionary history and conservation of the Brazilian Atlantic Forest. Heredity 106: 46-57.

Rodrigues ASL, Andelman SJ, Bakarr MI, Boitani L, Brooks TM, Cowling RM, Fishpool LDC, Fonseca GAB, Gaston KJ, Hoffmann M, Long JS, Marquet PA, Pilgrim JD, Pressey RL, Schipper J, Sechrest W, Stuart SN, Underhill LG, Waller RW, Watts MEJ \& Yan X (2004) Effectiveness of the global protected area network in representing species biodiversity. Nature 428: 640-643.

Schatz GE (2002) Taxonomy and herbaria in service of plant conservation: lessons from Madagascar's endemic families. Annals of the Missouri Botanical Garden 89: 145-152.

SMA - Secretaria do Estado do Meio Ambiente de São Paulo (2016) Publica a segunda revisão da lista oficial das espécies da flora ameaçadas de extinção no estado de São Paulo. Resolução n ${ }^{\circ} 57$, de 5 de junho de 2016. Disponível em <https://smastr16. blob.core.windows.net/legislacao/2016/12/ Resolu\%C3\%A7\%C3\%A3o-SMA-057-2016subst-300616.pdf>. Acesso em 4 dezembro 2016.

Souza VC, Mamede MCH, Cordeiro I, Prado J, Barros F, Wanderley MGL, Kageyama PY, Ceccantini G \& Rando JG (2007) Livro vermelho das espécies vegetais ameaçadas de extinção no estado de São Paulo. Instituto de Botânica, São Paulo. Pp. 15-20.

Tabarelli M, Pinto LP, Silva JMC, Hirota MM \& Bedê LC (2005) Desafios e oportunidades para a conservação da biodiversidade na Mata Atlântica brasileira. Megadiversidade 1: 132-138.

Tambosi LR, Martensen AC, Ribeiro MC \& Metzger JP (2014) A framework to optimize biodiversity restoration efforts based on habitat amount and landscape connectivity. Restoration Ecology 22: 169-177.

Ulloa-Ulloa CU, Acevedo-Rodríguez P, Beck S, Belgrano MJ, Bernal R, Berry PE, Brako L, Celis M, Davidse G, Forzza RC, Gradstein SR, Hokche O, León B, León-Yánez S, Magill RE, Neill DA, Nee M, Raven PH, Stimmel H, Strong MT, Villaseñor JL, Zarucchi JL, Zuloaga FO \& Jørgensen PM (2017) An integrated assessment of the vascular plant species of the Americas. Science 358: 1614-1617.

Wanderley MDGL, Shepherd GJ, Melhem TSA, Giulietti AM, Martins SE \& Kirizawa M (2005) Flora fanerogâmica do estado de São Paulo. Instituto de Botânica, São Paulo. Vol. 4, 437p.

Watson JEM, Dudley N, Segan DB \& Hockings M (2014) The performance and potential of protected areas. Nature 515: 67-73.

Weigrand-Junior R, Silva DC \& Silva DO (2011) Metas de Aichi: situação atual no Brasil. UICN, WWFBrasil e IPÊ, Brasília. 73p. 